\title{
Electromagnetic couplings of the chiral perturbation theory Lagrangian from the perturbative chiral quark model
}

\author{
V. E. Lyubovitskij *, Th. Gutsche ${ }^{*}$, Amand Faessler ${ }^{*}$ and R. Vinh Mau ${ }^{\dagger}$ \\ * Institut für Theoretische Physik, Universität Tübingen, \\ Auf der Morgenstelle 14, D-72076 Tübingen, Germany \\ $\dagger$ Laboratoire de Physique Nucleaire et des Hautes Energies, \\ Université P. et M. Curie, 4 Place Jussieu, 75252 Paris Cedex 05, France
}

\begin{abstract}
We apply the perturbative chiral quark model to the study of the low-energy $\pi N$ interaction. Using an effective chiral Lagrangian we reproduce the Weinberg-Tomozawa result for the $S$-wave $\pi N$ scattering lengths. After inclusion of the photon field we give predictions for the electromagnetic $O\left(p^{2}\right)$ lowenergy couplings of the chiral perturbation theory effective Lagrangian that define the electromagnetic mass shifts of nucleons and first-order $\left(e^{2}\right)$ radiative corrections to the $\pi N$ scattering amplitude. Finally, we estimate the leading isospin-breaking correction to the strong energy shift of the $\pi^{-} p$ atom in the $1 s$ state, which is relevant for the experiment "Pionic Hydrogen" at PSI.
\end{abstract}

PACS: 11.10.Ef, 12.39.Fe, 12.39.Ki, 13.40.Dk, 13.40.Ks, 14.20.Dh

Keywords: Chiral symmetry; Relativistic quark model; Relativistic effective Lagrangian; Pion-nucleon amplitude; Hadronic atoms.

\section{INTRODUCTION}

Hadron models set up to understand the structure of the nucleon should respect the constraints imposed by chiral symmetry. Spontaneous and explicit chiral symmetry breaking requires the existence of the pion whose mass vanishes in the limit of zero current quark mass. In turn, nucleon observables have to receive contributions from the pion cloud.

We recently suggested [1] a baryon model as based on earlier consideration [2], the perturbative chiral quark model (PCQM), which includes relativistic quark wave functions and confinement as well as the chiral symmetry requirements. The PCQM was successfully applied to $\sigma$-term physics [1] and extended to the study of electromagnetic properties of the nucleon. Similar perturbative quark models have also been studied in [3]. Although the Lagrangian for this model fulfils the $S U(2) \times S U(2)$ symmetry, it is in general nontrivial 
to identify the explicit hadron dynamics which leads to well-known predictions of current algebra for various observables. For instance, the $S$-wave $\pi N$ scattering at threshold is such a process in the presence of a pion cloud.

As was shown by Weinberg and Tomozawa [4.5], a model-independent expression for the $S$-wave $\pi N$ scattering lengths in terms of the pion mass and the weak decay constant is obtained when using the current algebra relations and the partial conservation of axial current (PCAC) assumption:

$$
a_{\frac{1}{2}}=\frac{M_{\pi}}{4 \pi F_{\pi}^{2}}+o\left(M_{\pi}\right) \quad \text { and } \quad a_{\frac{3}{2}}=-\frac{M_{\pi}}{8 \pi F_{\pi}^{2}}+o\left(M_{\pi}\right)
$$

To reproduce this result for the $\pi N$ scattering lengths one can also use a specific Lagrangian in the nucleon field refered to as the Weinberg-Tomozawa (WT) term [6]- [10]. Formulated on the quark level [11, 12] the WT term is part of the effective Weinberg Lagrangian [6]. This form can be derived from the original $\sigma$-model [13] by performing a chiral-field dependent rotation on the quark field. Thereby, the transformation eliminates the nonderivative coupling of the chiral (pion) field with the quarks and replaces it by a nonlinear derivative coupling (axial vector term + WT term + higher order terms in the chiral field). Both realizations of chirally-symmetric Lagrangians (the original $\sigma$-model and the Weinberg type Lagrangian) should á priori give the same result for the $\pi N S$-wave scattering lengths.

Starting point of present considerations is the PCQM [1] which, as dictated by global chiral symmetry, originally employs a nonderivative coupling between the pions and the quarks [13]. The purpose of the present work is first to demonstrate how, in the context of this chiral quark model, the WT result can be consistently obtained to the order of accuracy we are working in. Second, after fulfilling the constraint of reproducing $\pi N S$ wave scattering at threshold, we include the photon field in our formalism. We determine first-order $\left(e^{2}\right)$ radiative corrections to the nucleon mass and the pion-nucleon amplitude at threshold. We thereby predict the full set of $O\left(p^{2}\right)$ electromagnetic low-energy couplings (LECs) originally defined in the effective Lagrangian of chiral perturbation theory (ChPT). Quantitative information about these constants is important for the ongoing experimental and theoretical analysis of decay properties of the $\pi^{-} p$ atom (for a detailed discussion see Ref. [14]). In particular, we give a prediction for the leading isospin-breaking correction to the strong energy shift of the $\pi^{-} p$ atom in the $1 s$ state.

\section{PERTURBATIVE CHIRAL QUARK MODEL AND $\pi N S$-WAVE SCATTERING}

The PCQM is based on the effective, chirally invariant Lagrangian $\mathcal{L}_{i n v}$ [1]

$$
\begin{aligned}
\mathcal{L}_{i n v}(x) & =\bar{\psi}(x)\left\{i \not \partial-\gamma^{0} V(r)-S(r)\left[\frac{U+U^{\dagger}}{2}+\gamma^{5} \frac{U-U^{\dagger}}{2}\right]\right\} \psi(x) \\
& +\frac{F^{2}}{4} \operatorname{Tr}\left[\partial_{\mu} U \partial^{\mu} U^{\dagger}\right]
\end{aligned}
$$

where $\psi$ is the quark field, $U$ is the chiral field and $F=88 \mathrm{MeV}$ is the pion decay constant in the chiral limit [1:7]. The quarks move in a self-consistent field, represented by scalar $S(r)$ 
and vector $V(r)$ components of a static potential with $r=|\vec{x}|$ providing confinement. The interaction of quarks with Goldstone bosons is introduced via the chiral field $U=\exp [i \hat{\Phi} / F]$ which represents the exponentional parametrization of the nonlinear $\sigma$-model [13]. $\hat{\Phi}$ is the matrix of pseudoscalar mesons (in the following we restrict ourselves to the $S U(2)$ flavor case, $\hat{\Phi} \rightarrow \hat{\pi}=\vec{\pi} \vec{\tau}$.). For small fluctuations of the meson fields, one can use the expansion in powers of the parameter $1 / F$.

Usually, the Lagrangian (2) is linearized with respect to the field $\hat{\Phi}$ :

$$
U=\exp \left[i \frac{\hat{\Phi}}{F}\right] \simeq 1+i \frac{\hat{\Phi}}{F}+o\left(\frac{\hat{\Phi}}{F}\right) .
$$

Such an approximation is meaningful if we consider processes without external pions field (e.g. nucleon mass shift due to the pion cloud) or with a single external pion field (e.g. pion-nucleon form factor). The resulting approximate chiral invariance of the linearized Lagrangian [1, 11] guarantees both a conserved axial current (or PCAC in the presence of the meson mass term) and the Goldberger-Treiman relation [1, 11].

However, to calculate the pion-nucleon scattering amplitude, for consistency, we have to expand the invariant Lagrangian (2) up to quadratic terms in the pion field. Adding the mass term for pions we obtain the effective Lagrangian $\mathcal{L}_{\text {eff }}$ :

$$
\begin{aligned}
\mathcal{L}_{\text {eff }}(x) & =\mathcal{L}_{0}(x)+\mathcal{L}_{I}^{s t r}(x)+o\left(\vec{\pi}^{2}\right), \\
\mathcal{L}_{0}(x) & =\bar{\psi}(x)\left\{i \not \partial-S(r)-\gamma^{0} V(r)\right\} \psi(x)-\frac{1}{2} \vec{\pi}(x)\left(\square+M_{\pi}^{2}\right) \vec{\pi}(x), \\
\mathcal{L}_{I}^{s t r}(x) & =-\frac{S(r)}{F} \bar{\psi}(x) i \gamma^{5} \vec{\tau} \vec{\pi}(x) \psi(x)+\frac{S(r)}{2 F^{2}} \bar{\psi}(x) \vec{\pi}^{2}(x) \psi(x) .
\end{aligned}
$$

where $\square=\partial^{\mu} \partial_{\mu}$ and $M_{\pi}$ is the pion mass.

The quark field $\psi$ we expand in the basis of potential eigenstates as

$$
\begin{aligned}
\psi(x) & =\sum_{\alpha} b_{\alpha} u_{\alpha}(x)+\sum_{\beta} d_{\beta}^{\dagger} v_{\beta}(x), \\
u_{\alpha}(x) & =u_{\alpha}(\vec{x}) \exp \left(-i \mathcal{E}_{\alpha} t\right), \quad v_{\beta}(x)=v_{\beta}(\vec{x}) \exp \left(i \mathcal{E}_{\beta} t\right) .
\end{aligned}
$$

The sets of quark $\left\{u_{\alpha}\right\}$ and antiquark $\left\{v_{\beta}\right\}$ wave functions in orbits $\alpha$ and $\beta$ are solutions of the static Dirac equation with

$$
\begin{array}{ll} 
& {\left[-i \vec{\gamma} \vec{\nabla}+S(r)+\gamma^{0}\left(V(r)-\mathcal{E}_{\alpha}\right)\right] u_{\alpha}(\vec{x})=0} \\
\text { and } \quad\left[-i \vec{\gamma} \vec{\nabla}+S(r)+\gamma^{0}\left(V(r)+\mathcal{E}_{\beta}\right)\right] v_{\beta}(\vec{x})=0 .
\end{array}
$$

The wave functions satisfy to the normalization

$$
\int d^{3} x u_{\alpha^{\prime}}^{\dagger}(\vec{x}) u_{\alpha}(\vec{x})=\delta_{\alpha \alpha^{\prime}}, \quad \int d^{3} x v_{\beta^{\prime}}^{\dagger}(\vec{x}) v_{\beta}(\vec{x})=\delta_{\beta \beta^{\prime}},
$$

and completeness conditions

$$
\sum_{\alpha} u_{\alpha}(\vec{x}) u_{\alpha}^{\dagger}(\vec{y})+\sum_{\beta} v_{\beta}(\vec{x}) \bar{v}_{\beta}^{\dagger}(\vec{y})=\delta^{(3)}(\vec{x}-\vec{y}) .
$$


The expansion coefficients $b_{\alpha}$ and $d_{\beta}^{\dagger}$ are the corresponding single quark annihilation and antiquark creation operators, which fulfil the usual canonical anticommutation relations

$$
\left\{b_{\alpha}, b_{\alpha^{\prime}}^{\dagger}\right\}=\delta_{\alpha \alpha^{\prime}} \quad \text { and } \quad\left\{d_{\beta}, d_{\beta^{\prime}}^{\dagger}\right\}=\delta_{\beta \beta^{\prime}}
$$

The quark propagator in the binding potential is:

$$
\begin{aligned}
i G_{\psi}(x, y) & =<0|T\{\psi(x) \bar{\psi}(y)\}| 0> \\
& =\sum_{\alpha} u_{\alpha}(x) \bar{u}_{\alpha}(y) \theta\left(x_{0}-y_{0}\right)-\sum_{\beta} v_{\beta}(x) \bar{v}_{\beta}(y) \theta\left(y_{0}-x_{0}\right) .
\end{aligned}
$$

In the PCQM the strong pion-nucleon amplitude is defined ast

$$
{ }^{N}\left\langle\phi_{0} ; \pi_{j}\left|\sum_{n=1}^{2} \frac{i^{n}}{n !} \int d^{4} x_{1} \ldots \int d^{4} x_{n} T\left[\mathcal{L}_{I}^{s t r}\left(x_{1}\right) \ldots \mathcal{L}_{I}^{s t r}\left(x_{n}\right)\right]\right| \phi_{0} ; \pi_{i}\right\rangle_{c}^{N}
$$

where the state vector $\left|\phi_{0} ; \pi_{i}\right\rangle$ corresponds to the three quark ground state $\phi_{0}$ and a pion. Subscript "c" in Eq. (11) refers to contributions from connected graphs only. Superscript " $N$ " indicates that the matrix elements have to be projected onto the respective nucleon states. These nucleon states are conventionally set up by the product of single quark $S U(6)$ spin-flavor and $S U(3)_{c}$ color wave functions (see details in [16]), where the nonrelativistic single quark spin wave function is replaced by the relativistic ground state solution. At the tree level approximation (when we neglect pion loops) three diagrams (Fig.1) contribute to the $\pi N$ scattering amplitude: the $s$-channel pole (Fig.1a), the $u$-channel pole (Fig.1b) and the seagull diagram (Fig.1c). Pole diagrams are generated by the $\pi$-quark pseudoscalar coupling, whereas the seagull diagram is due to the quadratic term in the pion field.

To evaluate the matrix element (11) we use a set of identities deduced from the Dirac equation (6) and the completeness condition (8):

$$
\bar{\psi}(x) i \gamma^{5} S(|\vec{x}|) \psi(x)=\frac{1}{2} \partial_{\mu}^{x}\left\{\bar{\psi}(x) \gamma^{\mu} \gamma^{5} \psi(x)\right\}
$$

and

$$
\bar{\psi}(x) i \gamma^{5} S(|\vec{x}|) i G_{\psi}(x, y)=\frac{1}{2} \partial_{\mu}^{x}\left\{\bar{\psi}(x) \gamma^{\mu} \gamma^{5} i G_{\psi}(x, y)\right\}+\frac{1}{2} \bar{\psi}(x) \gamma^{5} \delta^{(4)}(x-y)
$$

Using Eq. (13) we obtain the key identity for the evaluation of Eq. (11):

$$
\begin{aligned}
& 4 \bar{\psi}(x) i \gamma^{5} S(|\vec{x}|) \tau_{i} i G_{\psi}(x, y) i \gamma^{5} S(|\vec{y}|) \tau_{j} \psi(y)=2 i \delta^{(4)}(x-y) \bar{\psi}(x) \tau_{i} \tau_{j} S(|\vec{x}|) \psi(x) \\
+ & \partial_{\mu}^{x}\left[\delta^{(4)}(x-y) \bar{\psi}(x) \gamma^{\mu} \tau_{i} \tau_{j} \psi(x)\right]+\partial_{\mu}^{x} \partial_{\nu}^{y}\left[\bar{\psi}(x) \gamma^{\mu} \gamma^{5} \tau_{i} i G_{\psi}(x, y) \gamma^{\nu} \gamma^{5} \tau_{j} \psi(y)\right] .
\end{aligned}
$$

With help of Eq. (14) the contributions to the $\pi N$ amplitude from the pole (PL) diagrams (Fig.1a and Fig.1b) are given by:

\footnotetext{
${ }^{1}$ Here and in the following we use the interaction Lagrangian and Wick's T-ordering in the calculation of matrix elements.
} 


$$
\begin{aligned}
& 2 \pi i \delta\left(p_{1}^{0}-p_{2}^{0}\right) \chi_{N_{f^{\prime}}}^{\dagger} T_{\pi N}^{i j ; \mathrm{PL}} \chi_{N_{f}}={ }^{N}\left\langle\phi_{0} ; \pi_{j}\left|\sum_{I=1}^{3} \hat{O}_{I}\right| \phi_{0} ; \pi_{i}\right\rangle^{N} \\
& \hat{O}_{1}=-\frac{i}{4 F^{2}} \int d^{4} x \bar{\psi}(x) \gamma^{\mu}\left[\vec{\pi}(x) \times \partial_{\mu} \vec{\pi}(x)\right] \vec{\tau} \psi(x) \\
& \hat{O}_{2}=-\frac{1}{4 F^{2}} \int d^{4} x \int d^{4} y \bar{\psi}(x) \gamma^{\mu} \gamma^{5} \partial_{\mu} \hat{\pi}(x) i G_{\psi}(x, y) \gamma^{\nu} \gamma^{5} \partial_{\nu} \hat{\pi}(y) \psi(y) \\
& \hat{O}_{3}=-\frac{i}{2 F^{2}} \int d^{4} x \bar{\psi}(x) S(|\vec{x}|) \vec{\pi}^{2} \psi(x) .
\end{aligned}
$$

where $\chi_{N_{f}}$ and $\chi_{N_{f^{\prime}}}^{\dagger}$ are the nucleon isospin wave functions in the initial and final states, $p_{1}^{0}$ and $p_{2}^{0}$ are the energies of incoming and outcoming pion, respectively. The seagull (SG) graph results in the expression

$$
2 \pi i \delta\left(p_{1}^{0}-p_{2}^{0}\right) \chi_{N_{f^{\prime}}}^{\dagger} T_{\pi N}^{i j ; \mathrm{SG}} \chi_{N_{f}}=-{ }^{N}\left\langle\phi_{0} ; \pi_{j}\left|\hat{O}_{3}\right| \phi_{0} ; \pi_{i}\right\rangle^{N}
$$

which cancels the corresponding term in Eq. (15). The sum of the pole and seagull diagrams is then:

$$
\begin{aligned}
2 \pi i \delta\left(p_{1}^{0}-p_{2}^{0}\right) \chi_{N_{f^{\prime}}}^{\dagger} T_{\pi N}^{i j} \chi_{N_{f}} & =2 \pi i \delta\left(p_{1}^{0}-p_{2}^{0}\right) \chi_{N_{f^{\prime}}}^{\dagger}\left[T_{\pi N}^{i j ; \mathrm{PL}}+T_{\pi N}^{i j ; \mathrm{SG}}\right] \chi_{N_{f}} \\
& ={ }^{N}\left\langle\phi_{0} ; \pi_{j}\left|\hat{O}_{1}+\hat{O}_{2}\right| \phi_{0} ; \pi_{i}\right\rangle^{N} .
\end{aligned}
$$

which is identical to the pion-nucleon amplitude generated by the Weinberg Lagrangian. The $\pi N$ scattering amplitude is:

$$
T_{\pi N}^{i j}=T_{\pi N}^{i j ; \mathrm{PL}}+T_{\pi N}^{i j ; \mathrm{SG}}=-\frac{i \varepsilon^{i j k}}{2 F^{2}} \tau^{k} M_{\pi}+O\left(\frac{\vec{p}^{2}}{M_{\pi}}, M_{\pi}^{2}\right),
$$

where $O\left(\vec{p}^{2} / M_{\pi}, M_{\pi}^{2}\right)$ represents a contribution which vanishes at threshold (with $\pi$ threemomentum $|\vec{p}| \rightarrow 0$ ) and faster than $M_{\pi}$. We, therefore, recover the result of Weinberg and Tomozawa for the $S$-wave $\pi N$ scattering lengths (11). Note, that only intermediate antiquark states contribute to the $\pi N$ amplitude at threshold, whereas the quark contribution (as in a relativistic theory with a fully covariant quark propagator) is vanishing. Also, due to the normalization (7) and the completeness condition (8), the explicit form of the quark and antiquark solutions is not needed in the derivation of (18).

Actually, one can generate the Weinberg-Tomozawa term at the Lagrangian level through the unitary chiral transformation $\psi \rightarrow \exp \left\{-i \gamma^{5} \hat{\Phi} /(2 F)\right\} \psi$ on the quark field $\psi$, as done, for example, in the cloudy bag model [11]. With this unitary chiral rotation the Lagrangian (2) transforms into a Weinberg-type form $\mathcal{L}^{W}$

$$
\begin{aligned}
\mathcal{L}^{W}(x) & =\mathcal{L}_{0}(x)+\mathcal{L}_{I}^{W ; s t r}(x)+o\left(\vec{\pi}^{2}\right), \\
\mathcal{L}_{0}(x) & =\bar{\psi}(x)\left\{i \not \partial-S(r)-\gamma^{0} V(r)\right\} \psi(x)-\frac{1}{2} \vec{\pi}(x)\left(\square+M_{\pi}^{2}\right) \vec{\pi}(x), \\
\mathcal{L}_{I}^{W ; s t r}(x) & =\frac{1}{2 F} \partial_{\mu} \vec{\pi}(x) \bar{\psi}(x) \gamma^{\mu} \gamma^{5} \vec{\tau} \psi(x)-\frac{\varepsilon_{i j k}}{4 F^{2}} \pi_{i}(x) \partial_{\mu} \pi_{j}(x) \bar{\psi}(x) \gamma^{\mu} \tau_{k} \psi(x),
\end{aligned}
$$

which contains now an axial vector coupling and the WT term. Thereby $\mathcal{L}_{I}^{W ; s t r}$ is the $O\left(\pi^{2}\right)$ strong interaction Lagrangian. Since the Lagrangians $\mathcal{L}_{\text {inv }}$ and $\mathcal{L}^{W}$ (up to order $1 / F^{2}$ ) are 
connected by a unitary transformation, both have to result in an identical expression for the $\pi N$ scattering amplitude, when evaluated up to a given order in $1 / F$. To make the connection explicit we start from the original Lagrangian (2). We show that the WeinbergTomozawa result can be reproduced if: i) we use the expansion of the chiral field up to quadratic terms and ii) we employ the full quark propagator including the antiquark components.

Thus we have demonstrated explicitly for the $\pi N$ amplitude up to order $\left(1 / F^{2}\right)$ that the two effective theories, the one involving the pseudoscalar coupling and the Weinberg type, are formally equivalent, both on the level of the Lagrangians and for the matrix elements. This equivalence is based on the unitary transformation of the quark fields, when, in addition, the quarks remain on their energy shell. The same relation also holds in a fully covariant formalism, when quarks/baryons are on their mass shell. Having set the basis in the strong interaction sector we will indicate in the next section that the two forms of the interaction Lagrangian also yield the same results when including the photon field.

\section{APPLICATIONS: ELECTROMAGNETIC CORRECTIONS TO THE NUCLEON MASS AND THE $\pi N$ AMPLITUDE AT THRESHOLD}

We can now use the equivalence between Eq. (44) and (19) to introduce electromagnetic corrections in the PCQM. This is performed in an unambiguous way into Eq. (舟:

$$
\partial_{\mu} \psi \rightarrow D_{\mu} \psi=\partial_{\mu} \psi+i e Q A_{\mu} \psi, \quad \partial_{\mu} \pi_{i} \rightarrow D_{\mu} \pi_{i}=\partial_{\mu} \pi_{i}+e \varepsilon_{3 i j} A_{\mu} \pi_{j}
$$

where $A_{\mu}$ is the electromagnetic field and $Q$ is the quark charge matrix. Following the Gell-Mann and Low theorem [17] the electromagnetic mass shift $\Delta m_{N}^{e m}$ of the nucleon with respect to the three-quark ground state $\left|\phi_{0}\right\rangle^{N}$ is given by

$$
\Delta m_{N}^{e m} \doteq{ }^{N}\left\langle\phi_{0}\left|-\frac{i}{2} \int \delta\left(x^{0}\right) d^{4} x \int d^{4} y T\left[\mathcal{L}^{e m}(x) \mathcal{L}^{e m}(y)\right]\right| \phi_{0}\right\rangle_{c}^{N}
$$

to order $e^{2}$ in the electromagnetic interaction. Subscript "c" in Eq. (21) refers to contributions from connected graphs only. With the quark-photon interaction defined by the Lagrangian

$$
\mathcal{L}^{e m}(x)=-e A_{\mu} \bar{\psi}(x) Q \gamma^{\mu} \psi(x)
$$

the electromagnetic mass shift $\Delta m_{N}^{e m}$ is generated by two diagrams: one-body (Fig.2a) and two-body diagram (Fig.2b).

The $\pi N$ scattering amplitude at threshold including the leading electromagnetic corrections (up to order $e^{2} / F^{2}$ ) is generated by the interaction Lagrangian

$$
\mathcal{L}_{I}(x)=\mathcal{L}_{I}^{s t r}(x)+\mathcal{L}_{I}^{e m}(x) .
$$

The strong interaction part $\mathcal{L}_{I}^{s t r}$ is already defined in Eq. (4) and $\mathcal{L}_{I}^{e m}$ is the $O(e)$ electromagnetic Lagrangian describing the interaction of quarks and pions with the electromagnetic field

$$
\mathcal{L}_{I}^{e m}=-e A_{\mu}(x) \bar{\psi}(x) Q \gamma^{\mu} \psi(x)-e \varepsilon_{3 i j} A_{\mu}(x) \pi_{i}(x) \partial^{\mu} \pi_{j}(x)
$$


The $\pi N$ amplitude in the presence of $O\left(e^{2}\right)$ radiative corrections is given by

$$
{ }^{N}\left\langle\phi_{0} ; \pi_{j}\left|\sum_{n=1}^{4} \frac{i^{n}}{n !} \int d^{4} x_{1} \ldots \int d^{4} x_{n} T\left[\mathcal{L}_{I}\left(x_{1}\right) \ldots \mathcal{L}_{I}\left(x_{n}\right)\right]\right| \phi_{0} ; \pi_{i}\right\rangle_{c}^{N} .
$$

Using Eq. (13) we obtain the identities

$$
\begin{aligned}
& 2 i G_{\psi}(x, y) i \gamma^{5} S(|\vec{y}|) \tau_{i} i G_{\psi}(y, z) \\
= & \delta^{(4)}(x-y) \gamma^{5} \tau_{i} i G_{\psi}(y, z)+\delta^{(4)}(y-z) i G_{\psi}(x, y) \gamma^{5} \tau_{i}+\partial_{\mu}^{y} \Gamma_{i}^{\mu}(x, y, z)
\end{aligned}
$$

and

$$
\begin{aligned}
& 4 i G_{\psi}(x, y) i \gamma^{5} S(|\vec{y}|) \tau_{i} i G_{\psi}(y, z) i \gamma^{5} S(|\vec{z}|) \tau_{j} i G_{\psi}(z, w) \\
= & 2 i \delta^{(4)}(y-z) i G_{\psi}(x, y) \tau_{i} \tau_{j} S(|\vec{y}|) i G_{\psi}(z, w)+\delta^{(4)}(x-y) \delta^{(4)}(z-w) \gamma^{5} \tau_{i} i G_{\psi}(x, w) \gamma^{5} \tau_{j} \\
+ & \delta^{(4)}(x-y) \delta^{(4)}(y-z) \tau_{i} \tau_{j} i G_{\psi}(x, w)+\delta^{(4)}(y-z) \delta^{(4)}(z-w) i G_{\psi}(x, w) \tau_{i} \tau_{j} \\
+ & \delta^{(4)}(z-w) \partial_{\mu}^{y} \Gamma_{i}^{\mu}(x, y, z) \gamma^{5} \tau_{j}+\delta^{(4)}(x-y) \gamma^{5} \tau_{i} \partial_{\mu}^{z} \Gamma_{j}^{\mu}(x, z, w)+\partial_{\mu}^{y} \partial_{\nu}^{z} \Gamma_{i j}^{\mu \nu}(x, y, z, w)
\end{aligned}
$$

where

$$
\begin{aligned}
\Gamma_{i}^{\mu}(x, y, z) & =i G_{\psi}(x, y) \gamma^{\mu} \gamma^{5} \tau_{i} i G_{\psi}(y, z) \\
\Gamma_{i j}^{\mu \nu}(x, y, z, w) & =i G_{\psi}(x, y) \gamma^{\mu} \gamma^{5} \tau_{i} i G_{\psi}(y, z) \gamma^{\nu} \gamma^{5} \tau_{j} i G_{\psi}(z, w) .
\end{aligned}
$$

With help of Eqs. (26) and (27) one can show that the matrix element (25) is identical to the one where $\mathcal{L}_{I}$ is replaced by the Weinberg interaction Lagrangian including photons $\mathcal{L}_{I}^{W}$

$$
\mathcal{L}_{I}^{W}(x)=\mathcal{L}_{I}^{W ; s t r}(x)+\mathcal{L}_{I}^{W ; e m}(x)
$$

where $\mathcal{L}_{I}^{W ; s t r}$ is given in Eq. (19) and the additional electromagnetic part $\mathcal{L}_{I}^{W ; e m}$ is given by

$$
\begin{aligned}
\mathcal{L}_{I}^{W ; e m}(x) & =\mathcal{L}_{I}^{e m}(x)+\frac{e \varepsilon_{3 i j}}{2 F} A_{\mu}(x) \pi_{j}(x) \bar{\psi}(x) \gamma^{\mu} \gamma^{5} \tau_{i} \psi(x) \\
& +\frac{e}{4 F^{2}} A_{\mu}(x) \bar{\psi}(x) \gamma^{\mu}\left[\vec{\pi}^{2}(x) \tau_{3}-\vec{\pi}(x) \vec{\tau} \pi^{0}(x)\right] \psi(x)
\end{aligned}
$$

We therefore have the key identity

$$
\begin{aligned}
& { }^{N}\left\langle\phi_{0} ; \pi_{j}\left|\sum_{n=1}^{4} \frac{i^{n}}{n !} \int d^{4} x_{1} \ldots \int d^{4} x_{n} T\left[\mathcal{L}_{I}\left(x_{1}\right) \ldots \mathcal{L}_{I}\left(x_{n}\right)\right]\right| \phi_{0} ; \pi_{i}\right\rangle_{c}^{N} \\
= & { }^{N}\left\langle\phi_{0} ; \pi_{j}\left|\sum_{n=1}^{4} \frac{i^{n}}{n !} \int d^{4} x_{1} \ldots \int d^{4} x_{n} T\left[\mathcal{L}_{I}^{W}\left(x_{1}\right) \ldots \mathcal{L}_{I}^{W}\left(x_{n}\right)\right]\right| \phi_{0} ; \pi_{i}\right\rangle_{c}^{N}
\end{aligned}
$$

extending the consistency between the two interaction Lagrangians to the case where photons are present. For the equivalence to hold is essential that the photons are introduced consistently in both formalisms, that is by minimal substitution. The diagrams for $O\left(e^{2} / F^{2}\right)$ radiative corrections to the $\pi N$ amplitude at threshold are shown in Fig.3. They are generated by the matrix element (31) with the Weinberg interaction Lagrangian $\mathcal{L}_{I}^{W}$. Again, 
the set of diagrams of Fig.3 can be identically deduced from the corresponding one generated by our original interaction Lagrangian $\mathcal{L}_{I}$. To evaluate the diagrams shown in Figs. 2 and 3 we use the photon propagator in the Coulomb gauge to separate the contributions from Coulomb $\left(A_{0}\right)$ and transverse $\left(A_{i}\right)$ photons. The propagators of Coulomb $\left(D_{00}\right)$ and transverse $\left(D_{i j}\right)$ photons are given by

$$
i D_{00}(x-y)=<0\left|T\left\{A_{0}(x) A_{0}(y)\right\}\right| 0>=i \int \frac{d^{4} k}{(2 \pi)^{4}} \frac{e^{-i k(x-y)}}{\vec{k}^{2}}
$$

and

$$
i D_{i j}(x-y)=<0\left|T\left\{A_{i}(x) A_{j}(y)\right\}\right| 0>=i \int \frac{d^{4} k}{(2 \pi)^{4}} \frac{e^{-i k(x-y)}}{k^{2}+i \varepsilon}\left\{\delta_{i j}-\frac{k_{i} k_{j}}{\vec{k}^{2}}\right\} .
$$

First, we analyze the electromagnetic mass shift of the nucleon $\left(\Delta m_{N}^{e m}\right)$. The contributions of diagrams Fig.2a (one-body term $\Delta m_{N}^{e m ; a}$ ) and Fig.2b (two-body term $\Delta m_{N}^{e m ; b}$ ) are given by

$$
\Delta m_{N}^{e m ; a}=e^{2} \cdot{ }^{N}\left\langle\phi_{0}\left|\int d^{4} x \int d^{4} y \delta\left(x^{0}\right) D_{\mu \nu}(x-y) \bar{\psi}_{0}(x) \gamma^{\mu} Q i G_{\psi}(x, y) \gamma^{\nu} Q \psi_{0}(y)\right| \phi_{0}\right\rangle^{N}
$$

and

$$
\Delta m_{N}^{e m ; b}=\frac{e^{2}}{2} \cdot{ }^{N}\left\langle\phi_{0}\left|\int d^{4} x \int d^{4} y \delta\left(x^{0}\right) D_{\mu \nu}(x-y) \bar{\psi}_{0}(x) \gamma^{\mu} Q \psi_{0}(x) \bar{\psi}_{0}(y) \gamma^{\nu} Q \psi_{0}(y)\right| \phi_{0}\right\rangle^{N}
$$

In the following we truncate the expansion of the quark propagator to the ground state eigen mode:

$$
i G_{\psi}(x, y) \rightarrow i G_{0}(x, y) \doteq u_{0}(\vec{x}) \bar{u}_{0}(\vec{y}) e^{-i \mathcal{E}_{\alpha}\left(x_{0}-y_{0}\right)} \theta\left(x_{0}-y_{0}\right)
$$

that is we restrict the intermediate baryon states to $N$ and $\Delta$ configurations. Inclusion of excited baryon states will be subject of future investigations. With the approximation (36), the one- and two-body contributions reduce to

$$
\Delta m_{N}^{e m ; a}=\frac{e^{2}}{16 \pi^{3}}\left\langle N\left|\sum_{i=1}^{3}\left(Q^{2}\right)^{(i)}\right| N\right\rangle \int \frac{d^{3} q}{\vec{q}^{2}}\left\{\left[G_{E}^{p}\left(-\vec{q}^{2}\right)\right]^{2}-\frac{\vec{q}^{2}}{2 m_{N}^{2}}\left[G_{M}^{p}\left(-\vec{q}^{2}\right)\right]^{2}\right\}
$$

and

$$
\begin{aligned}
\Delta m_{N}^{e m ; b} & =\frac{e^{2}}{16 \pi^{3}} \int \frac{d^{3} q}{\vec{q}^{2}}\left\{\left\langle N\left|\sum_{i \neq j}^{3} Q^{(i)} Q^{(j)}\right| N\right\rangle\left[G_{E}^{p}\left(-\vec{q}^{2}\right)\right]^{2}\right. \\
& \left.-\left\langle N\left|\sum_{i \neq j}^{3} Q^{(i)} Q^{(j)} \vec{\sigma}^{(i)} \vec{\sigma}^{(j)}\right| N\right\rangle \frac{\vec{q}^{2}}{6 m_{N}^{2}}\left[G_{M}^{p}\left(-\vec{q}^{2}\right)\right]^{2}\right\}
\end{aligned}
$$

\footnotetext{
${ }^{2}$ It can be shown that the results do not depend on the choice of the gauge.
} 
where $|N\rangle$ is the $\mathrm{SU}(6)$ spin-flavor w.f. of the nucleon. Here we introduce the proton charge $\left(G_{E}^{p}\right)$ and magnetic $\left(G_{M}^{p}\right)$ form factors calculated at zeroth order [1] (meson cloud corrections are not taken into account) with

$$
\chi_{N_{s^{\prime}}}^{\dagger} \chi_{N_{s}} G_{E}\left(-\vec{q}^{2}\right)={ }^{N}\left\langle\phi_{0}\left|\int d^{3} x \bar{\psi}_{0}(\vec{x}) \gamma^{0} \psi_{0}(\vec{x}) e^{i \vec{q} \vec{x}}\right| \phi_{0}\right\rangle^{N}
$$

and

$$
\chi_{N_{s^{\prime}}}^{\dagger} \frac{i\left[\vec{\sigma}_{N} \times \vec{q}\right]}{2 m_{N}} \chi_{N_{s}} G_{M}\left(-\vec{q}^{2}\right)={ }^{N}\left\langle\phi_{0}\left|\int d^{3} x \bar{\psi}_{0}(\vec{x}) \vec{\gamma} \psi_{0}(\vec{x}) e^{i \vec{q} \vec{x}}\right| \phi_{0}\right\rangle^{N}
$$

where $\chi_{N_{s}}$ and $\chi_{N_{s^{\prime}}}^{\dagger}$ are the nucleon spin w.f. in the initial and final state; $\vec{\sigma}_{N}$ is the nucleon spin operator. Note that the contributions of Coulomb and transverse photons to the electromagnetic mass shifts (see Eqs. (37) and (38)) are related to the nucleon charge and magnetic form factors, respectively. The sum of expectation values

$$
\left\langle N\left|\sum_{i=1}^{3}\left(Q^{2}\right)^{(i)}\right|\right\rangle N+\left\langle N\left|\sum_{i \neq j}^{3} Q^{(i)} Q^{(j)}\right| N\right\rangle=\left\{\begin{array}{l}
1 \text { for } N=p \\
0 \text { for } N=n
\end{array}\right.
$$

is equivalent to the charge matrix of nucleons $\left(Q_{N}\right.$ being the nucleon charge). In the limit $m_{N} \rightarrow \infty$ (when we neglect the contribution of $G_{M}^{p}$ in Eqs. (37) and (38)) we obtain for the electromagnetic mass shifts

$$
\Delta m_{N}^{e m}=\Delta m_{N}^{e m ; a}+\Delta m_{N}^{e m ; b}=\frac{\alpha Q_{N}^{2}}{4 \pi^{2}} \int \frac{d^{3} q}{\vec{q}^{2}}\left[G_{E}^{p}\left(-\vec{q}^{2}\right)\right]^{2}
$$

consistent with the result (Eq. (12.4)) of Ref. [18]. Hence, the electromagnetic mass shift of the neutron vanishes in the heavy nucleon limit.

In the numerical analysis we use the variational Gaussian ansatz [1, 19] for the quark ground state wave function with the following analytical form:

$$
u_{0}(\vec{x})=N \exp \left[-\frac{\vec{x}^{2}}{2 R^{2}}\right]\left(\begin{array}{c}
1 \\
i \rho \vec{\sigma} \vec{x} / R
\end{array}\right) \chi_{s} \chi_{f} \chi_{c}
$$

where $N=\left[\pi^{3 / 2} R^{3}\left(1+3 \rho^{2} / 2\right)\right]^{-1 / 2}$ is a constant fixed by the normalization condition $\int d^{3} x u_{0}^{\dagger}(x) u_{0}(x) \equiv 1 ; \chi_{s}, \chi_{f}, \chi_{c}$ are the spin, flavor and color quark wave functions, respectively. Our Gaussian ansatz contains two model parameters: the dimensional parameter $R$ and the dimensionless parameter $\rho$. The parameter $\rho$ can be related to the axial coupling constant $g_{A}$ calculated in the leading-order (or the three quark-core) approximation:

$$
g_{A}=\frac{5}{3}\left(1-\frac{2 \rho^{2}}{1+\frac{3}{2} \rho^{2}}\right)
$$

Solving this equation in $\rho$, one gets:

$$
\rho^{2}=2\left(\frac{1-\frac{3}{5} g_{A}}{1+\frac{9}{5} g_{A}}\right)
$$


The parameter $R$ can be physically understood as the mean radius of the three-quark core and is related to the charge radius $\left\langle r_{E}^{2}\right\rangle_{L O}^{P}$ of the proton in the leading-order (LO) approximation as

$$
\left\langle r_{E}^{2}\right\rangle_{L O}^{P}=\int d^{3} x u_{0}^{\dagger}(\vec{x}) \vec{x}^{2} u_{0}(\vec{x})=\frac{3 R^{2}}{2} \frac{1+\frac{5}{2} \rho^{2}}{1+\frac{3}{2} \rho^{2}} .
$$

In our calculations we use the value $g_{A}=1.25$ obtained in ChPT [7]. Therefore, we have only one free parameter, that is $R$. In the numerical studies [1] $\mathrm{R}$ is varied in the region from 0.55 fm to $0.65 \mathrm{fm}$, which corresponds to a change of $\left\langle r_{E}^{2}\right\rangle_{3 q-\text { core }}^{P}$ from 0.5 to $0.7 \mathrm{fm}^{2}$. The exact Gaussian ansatz (43) restricts the potentials $S(r)$ and $V(r)$ to a form proportional to $r^{2}$. They are expressed in terms of the parameters $R$ and $\rho$ (for details see Ref. [1]).

Using the Gaussian ansatz (43) the proton charge and magnetic form factors at zeroth order are determined as [1]:

$$
\begin{aligned}
& G_{E}\left(-\vec{q}^{2}\right)=\exp \left(-\frac{\vec{q}^{2} R^{2}}{4}\right)\left[1-\frac{\vec{q}^{2} R^{2}}{4} \kappa\right], \\
& G_{M}\left(-\vec{q}^{2}\right)=\exp \left(-\frac{\vec{q}^{2} R^{2}}{4}\right) 2 m_{N} R \sqrt{\kappa\left(1-\frac{3}{2} \kappa\right)}, \quad \kappa=\frac{1}{2}-\frac{3}{10} g_{A},
\end{aligned}
$$

which yield finally the electromagnetic mass shift

$$
\begin{aligned}
\Delta m_{p}^{e m} & =\frac{\alpha}{R \sqrt{2 \pi}}\left[1-\frac{\kappa}{2}+\frac{3}{16} \kappa^{2}-\frac{34}{9} \kappa\left(1-\frac{3}{2} \kappa\right)\right], \\
\Delta m_{n}^{e m} & =-\frac{\alpha}{R \sqrt{2 \pi}} \frac{8}{3} \kappa\left(1-\frac{3}{2} \kappa\right),
\end{aligned}
$$

where $\alpha=1 / 137$ is the fine structure coupling. For our set of parameters $g_{A}=1.25$ and $R=0.6 \pm 0.05 \mathrm{fm}$ we get $\Delta m_{p}^{e m}=0.54 \pm 0.04 \mathrm{MeV}, \Delta m_{n}^{e m}=-0.26 \pm 0.02 \mathrm{MeV}$ and $\Delta m_{n}^{e m}-\Delta m_{p}^{e m}=-0.8 \pm 0.06 \mathrm{MeV}$. The uncertainties of our results correspond to the variation of the parameter $R$. Our predictions are in qualitative agreement with the results obtained by Gasser and Leutwyler using the Cottingham formula [18]: $\Delta m_{p}^{e m}=0.63 \mathrm{MeV}$, $\Delta m_{n}^{e m}=-0.13 \mathrm{MeV}, \Delta m_{n}^{e m}-\Delta m_{p}^{e m}=-0.76 \mathrm{MeV}$.

To compare our prediction for the electromagnetic mass shifts of the nucleons with the result of ChPT [9], we recall the part of the ChPT Lagrangian [9] which is responsible for radiative corrections

$$
\mathcal{L}_{C h P T}^{e^{2}}=e^{2} \bar{N}\left\{f_{1}\left(1-\frac{\vec{\pi}^{2}-\left(\pi^{0}\right)^{2}}{F^{2}}\right)+\frac{f_{2}}{2}\left(\tau_{3}-\frac{\vec{\pi}^{2} \tau_{3}-\pi^{0} \vec{\pi} \vec{\tau}}{2 F^{2}}\right)+f_{3}\right\} N .
$$

The $O\left(p^{2}\right)$ low-energy constants (LECs) $f_{1}, f_{2}$ and $f_{3}$ contain the effect of the direct quarkphoton interaction. Matching our results for the nucleon mass shifts to the predictions of ChPT [9] with

$$
\left.\Delta m_{p}^{e m}\right|_{C h P T}=-4 \pi \alpha\left(f_{1}+f_{3}+\frac{f_{2}}{2}\right),\left.\Delta m_{n}^{e m}\right|_{C h P T}=-4 \pi \alpha\left(f_{1}+f_{3}-\frac{f_{2}}{2}\right)
$$

we obtain following relations for the coupling constants $f_{1}, f_{2}$ and $f_{3}$ : 


$$
\begin{gathered}
f_{2}=-\frac{1}{2 R(2 \pi)^{3 / 2}}\left[1-\frac{29}{18} \kappa+\frac{89}{48} \kappa^{2}\right], \\
f_{1}+f_{3}=-\frac{1}{4 R(2 \pi)^{3 / 2}}\left[1-\frac{125}{18} \kappa+\frac{473}{48} \kappa^{2}\right] .
\end{gathered}
$$

Our numerical result for $f_{2}=-8.7 \pm 0.7 \mathrm{MeV}$ is in good agreement with the value of $f_{2}=-8.3 \pm 3.3 \mathrm{MeV}$ 9,14 extracted from the analysis of the elastic electron scattering cross section using the Cottingham formula [18]. For $f_{1}+f_{3}$ we get $-1.5 \pm 0.1 \mathrm{MeV}$.

We now apply our model to the analysis of radiative corrections to the $\pi N$ amplitude at the lowest order in $e^{2}$, and to the determination of $f_{1}, f_{3}$ and $f_{1} / f_{2}$ separately. We denote the corresponding matrix element associated with the nucleon flavor transition $N_{1} \rightarrow N_{2}$ by $M_{N_{1} N_{2}}^{\left(e^{2}\right) ; j}$. In the Coulomb gauge only six diagrams (Fig.3a-3f) contribute to the radiative correction to the $\pi N$ amplitude at threshold. The contribution of the other diagrams (Fig.3g3o) vanishes. The contributions of the different diagrams of Fig.3 are as follow:

$$
\begin{aligned}
\left.M_{N_{1} N_{2}}^{\left(e^{2}\right) ; j}\right|_{a+b} & =-\frac{e^{2}}{F^{2}} \cdot{ }^{N}\left\langle\phi_{0}\right| \int d^{4} x \int d^{4} y D_{\mu \nu}(x-y) \bar{\psi}_{0}(x) \gamma^{\mu} \\
& \times\left(T^{i j} G_{\psi}(x, y) Q+Q G_{\psi}(x, y) T^{i j}\right) \gamma^{\nu} \psi_{0}(y)\left|\phi_{0}\right\rangle^{N}
\end{aligned}
$$

for Fig.3a and Fig.3b where $T^{i j}=2 \delta^{i j} \tau^{3}-\delta^{i 3} \tau^{j}-\delta^{j 3} \tau^{i}$,

$$
\begin{aligned}
\left.M_{N_{1} N_{2}}^{\left(e^{2}\right) ; i}\right|_{c} & =\frac{i e^{2}}{F^{2}} \cdot{ }^{N}\left\langle\phi_{0}\right| \int d^{4} x \int d^{4} y D_{\mu \nu}(x-y) \bar{\psi}_{0}(x) \gamma^{\mu} \\
& \times T^{i j} \psi_{0}(x) \bar{\psi}_{0}(y) \gamma^{\nu} Q \psi_{0}(y)\left|\phi_{0}\right\rangle^{N}
\end{aligned}
$$

for Figs.3c,

$$
\begin{aligned}
\left.M_{N_{1} N_{2}}^{\left(e^{2}\right) ; j}\right|_{d+e} & =-\frac{e^{2}}{F^{2}} \cdot{ }^{N}\left\langle\phi_{0}\right| \int d^{4} x \int d^{4} y D_{\mu \nu}(x-y) \bar{\psi}_{0}(x) \gamma^{\mu} \gamma^{5} \\
& \times\left(\varepsilon^{3 i k} \varepsilon^{3 j m}+\varepsilon^{3 j k} \varepsilon^{3 i m}\right) \tau^{k} G_{\psi}(x, y) \gamma^{\nu} \gamma^{5} \tau^{m} \psi_{0}(y)\left|\phi_{0}\right\rangle^{N}
\end{aligned}
$$

for Fig.3d and Fig.3e,

$$
\begin{aligned}
\left.M_{N_{1} N_{2}}^{\left(e^{2}\right) ; j}\right|_{f} & =\frac{i e^{2}}{F^{2}} \cdot{ }^{N}\left\langle\phi_{0}\right| \int d^{4} x \int d^{4} y D_{\mu \nu}(x-y) \bar{\psi}_{0}(x) \gamma^{\mu} \gamma^{5} \\
& \times \varepsilon^{3 i k} \varepsilon^{3 j m} \tau^{k} \psi_{0}(x) \bar{\psi}_{0}(y) \gamma^{\nu} \gamma^{5} \tau^{m} \psi_{0}(y)\left|\phi_{0}\right\rangle^{N}
\end{aligned}
$$

for Fig.3f.

Truncating the quark propagator to the ground state mode we obtain the total expression for the $O\left(e^{2}\right) \pi N$ scattering amplitude, at threshold:

$$
\begin{aligned}
M_{N_{1} N_{2}}^{\left(e^{2}\right) ; i j} & =\frac{\alpha}{(4 \pi F)^{2}} \int \frac{d^{3} q}{\vec{q}^{2}}\left[C_{N_{1} N_{2}}^{E ; i j} G_{E}^{2}\left(-\vec{q}^{2}\right)-\frac{\vec{q}^{2}}{2 m_{N}^{2}} C_{N_{1} N_{2}}^{M ; i j} G_{M}^{2}\left(-\vec{q}^{2}\right)\right. \\
& \left.+\frac{6}{25} \frac{d_{+}^{2}\left(\vec{q}^{2}\right)}{d_{-}^{2}\left(\vec{q}^{2}\right)} C_{N_{1} N_{2}}^{A ; i j} G_{A}^{2}\left(-\vec{q}^{2}\right)\right] .
\end{aligned}
$$

where $G_{A}$ is the axial charge form factor calculated at zeroth order [1] 


$$
\begin{aligned}
\left(\chi_{N_{s^{\prime}}}^{\dagger} \sigma_{N}^{3} \chi_{N_{s}}\right)\left(\chi_{N_{f^{\prime}}}^{\dagger} \tau_{N}^{3} \chi_{N_{f}}\right) G_{A}\left(-\vec{q}^{2}\right) & ={ }^{N}\left\langle\phi_{0}\left|\int d^{3} x \bar{u}_{0}(\vec{x}) \gamma^{3} \gamma^{5} \tau^{3} u_{0}(\vec{x}) e^{i \vec{q} \vec{x}}\right| \phi_{0}\right\rangle^{N}, \\
G_{A}\left(-\vec{q}^{2}\right) & =g_{A} \exp \left(-\frac{\vec{q}^{2} R^{2}}{4}\right) d_{-}\left(\vec{q}^{2}\right)
\end{aligned}
$$

and

$$
d_{ \pm}\left(\vec{q}^{2}\right)=1 \pm \frac{\vec{q}^{2} R^{2}}{4} \frac{\kappa}{1-2 \kappa} .
$$

Here $\tau_{N}^{3}$ is the nucleon isospin matrix. Thus, the contribution of the Coulomb photons to the amplitude $M_{N_{1} N_{2}}^{\left(e^{2}\right) i j}$ is parametrized by the proton charge form factor $\left(G_{E}\right)$, transverse photons are related to the proton magnetic $\left(G_{M}\right)$ and axial charge $\left(G_{A}\right)$ form factors.

The factors $C_{N_{1} N_{2}}^{E}, C_{N_{1} N_{2}}^{M}$ and $C_{N_{1} N_{2}}^{A}$ are the spin-flavor coefficients

$$
\begin{aligned}
C_{N_{1} N_{2}}^{E ; i j} & =<N_{1}\left|\sum_{m=1}^{3}\left(2 \delta^{i j}\left\{\tau^{3}, Q\right\}-\delta^{i 3}\left\{\tau^{j}, Q\right\}-\delta^{j 3}\left\{\tau^{i}, Q\right\}\right)^{(m)}\right| N_{2}> \\
& +2<N_{1}\left|\sum_{m \neq n}^{3}\left(2 \delta^{i j} \tau^{3}-\delta^{i 3} \tau^{j}-\delta^{j 3} \tau^{i}\right)^{(m)} Q^{(n)}\right| N_{2}>, \\
C_{N_{1} N_{2}}^{M ; i j} & =<N_{1}\left|\sum_{m=1}^{3}\left(2 \delta^{i j}\left\{\tau^{3}, Q\right\}-\delta^{i 3}\left\{\tau^{j}, Q\right\}-\delta^{j 3}\left\{\tau^{i}, Q\right\}\right)^{(m)}\right| N_{2}> \\
& +\frac{2}{3}<N_{1}\left|\sum_{m \neq n}^{3}\left(2 \delta^{i j} \tau^{3}-\delta^{i 3} \tau^{j}-\delta^{j 3} \tau^{i}\right)^{(m)} Q^{(n)} \vec{\sigma}^{(m)} \vec{\sigma}^{(n)}\right| N_{2}>, \\
C_{N_{1} N_{2}}^{A ; i j} & =6\left(\delta^{i j}-\delta^{i 3} \delta^{j 3}\right)<N_{1}\left|\left(\sum_{m=1}^{3} \mathrm{I}^{(m)}+\frac{1}{9} \sum_{m \neq n}^{3} \vec{\tau}^{(m)} \vec{\tau}^{(n)} \vec{\sigma}^{(m)} \vec{\sigma}^{(n)}\right)\right| N_{2}>,
\end{aligned}
$$

where the notation $\{\tau, Q\}$ denotes the anticommutator. The matrix elements of Eqs. (58)(60) can be easily evaluated by using the $S U(6)$ spin-flavor wave functions of the nucleon.

Finally the first-order radiative corrections to the $\pi N$ scattering amplitude at threshold are given by

$$
\begin{aligned}
T_{\pi N}^{\left(e^{2}\right) ; i j} & =-\frac{1}{(4 \pi)^{3}} \int \frac{d^{3} q}{\vec{q}^{2}}\left\{T_{f_{1}}^{i j}\left[\left[G_{E}^{p}\left(-\vec{q}^{2}\right)\right]^{2}-\frac{19 \vec{q}^{2}}{6 m_{N}^{2}}\left[G_{M}^{p}\left(-\vec{q}^{2}\right)\right]^{2}+\frac{114}{25} \frac{d_{+}^{2}\left(\vec{q}^{2}\right)}{d_{-}^{2}\left(\vec{q}^{2}\right)} G_{A}^{2}\left(-\vec{q}^{2}\right)\right]\right. \\
& \left.+T_{f_{2}}^{i j}\left[\left[G_{E}^{p}\left(-\vec{q}^{2}\right)\right]^{2}-\frac{5 \vec{q}^{2}}{18 m_{N}^{2}}\left[G_{M}^{p}\left(-\vec{q}^{2}\right)\right]^{2}\right]\right\} \\
& =-\frac{1}{8 R} \frac{1}{(2 \pi)^{3 / 2}}\left\{T_{f_{1}}^{i j}\left[\frac{41}{3}-\frac{115}{2} \kappa+\frac{953}{16} \kappa^{2}\right]+T_{f_{2}}^{i j}\left[1-\frac{29}{18} \kappa+\frac{89}{48} \kappa^{2}\right]\right\} .
\end{aligned}
$$

where

$$
T_{f_{1}}^{i j}=-\frac{8 \pi \alpha}{F^{2}}\left(\delta^{i j}-\delta^{i 3} \delta^{j 3}\right) \text { and } T_{f_{2}}^{i j}=-\frac{4 \pi \alpha}{F^{2}}\left(2 \delta^{i j} \tau^{3}-\delta^{i 3} \tau^{j}-\delta^{j 3} \tau^{i}\right) .
$$

Again, as in the case of electromagnetic mass shifts, the amplitude $M_{i n v}^{e^{2} \pi N}$ is gaugeindependent. In ChPT the corresponding amplitude is given by [9]

$$
\left.T_{\pi N}^{\left(e^{2}\right) ; j}\right|_{C h P T}=f_{1} T_{f_{1}}^{i j}+\frac{f_{2}}{4} T_{f_{2}}^{i j} .
$$


Comparing Eqs. (61) and (63) we get the same expression for $f_{2}$ as already obtained from the electromagnetic mass shift (51). We also deduce the following relations:

$$
\begin{aligned}
f_{1} & =-\frac{1}{8 R(2 \pi)^{3 / 2}}\left[\frac{41}{3}-\frac{115}{2} \kappa+\frac{953}{16} \kappa^{2}\right], \\
f_{3} & =\frac{1}{8 R(2 \pi)^{3 / 2}}\left[\frac{35}{3}-\frac{785}{18} \kappa+\frac{1913}{48} \kappa^{2}\right], \\
\frac{f_{1}}{f_{2}} & =\frac{\frac{41}{12}-\frac{115}{8} \kappa+\frac{953}{64} \kappa^{2}}{1-\frac{29}{18} \kappa+\frac{89}{48} \kappa^{2}} .
\end{aligned}
$$

The predicted ratio for $f_{1} / f_{2}$ depends on only one model parameter $\rho$ (or $\kappa$ ) which is related to the axial nucleon charge $g_{A}$ calculated at zeroth order (see Eq. (45)). In addition, the constants $f_{1}, f_{2}$ and $f_{3}$ depend on the size parameter $R$ of the bound quark. For our "canonical" set of parameters, $g_{A}=1.25$ and $R=0.6 \pm 0.05 \mathrm{fm}$, used in the calculations of nucleon electromagnetic form factors and meson-baryon sigma terms [1] we obtain:

$$
\begin{array}{ll}
f_{1}=-19.5 \pm 1.6 \mathrm{MeV}, & f_{2}=-8.7 \pm 0.7 \mathrm{MeV}, \\
f_{3}=18 \pm 1.5 \mathrm{MeV}, & \frac{f_{1}}{f_{2}}=2.2 .
\end{array}
$$

Using these values of $f_{1}$ and $f_{2}$ we can estimate the isospin-breaking correction to the energy shift of the $\pi^{-} p$ atom in the $1 s$ state. The strong energy-level shift $\epsilon_{1 s}$ of the $\pi^{-} p$ atom is given by the model-independent formula [14]:

$$
\epsilon_{1 s}=\epsilon_{1 s}^{L O}+\epsilon_{1 s}^{N L O}=\epsilon_{1 s}^{L O}\left(1+\delta_{\epsilon}\right) .
$$

where the leading order $(\mathrm{LO})$ or isospin-symmetric contribution is $\epsilon_{1 s}^{L O}$ and the next-toleading order (NLO) or isospin-breaking contribution is $\epsilon_{1 s}^{N L O}$. The quantity $\epsilon_{1 s}^{L O}$ is expressed with the help of the well-known Deser formula [20] in terms of the $S$-wave $\pi N$ scattering lengths $a_{\frac{1}{2}}$ and $a_{\frac{3}{2}}$ with

$$
\epsilon_{1 s}^{L O}=-2 \alpha^{3} \mu_{c}^{2} \mathcal{A}_{s t r} \quad \text { and } \quad \mathcal{A}_{s t r}=\frac{1}{3}\left(2 a_{\frac{1}{2}}+a_{\frac{3}{2}}\right) .
$$

The reduced mass of the $\pi^{-} p$ atom is denoted by $\mu_{c}=m_{p} M_{\pi^{+}} /\left(m_{p}+M_{\pi^{+}}\right)$and $\mathcal{A}_{\text {str }}=$ $(88.4 \pm 1.9) \times 10^{-3} M_{\pi^{+}}^{-1}$ is the strong (isospin-invariant) regular part of the $\pi^{-} p$ scattering amplitude at threshold [21] (for the definitions of these quantities see Ref. [14]). In ChPT the quantity $\delta_{\epsilon}$, the ratio of NLO to LO corrections, is expressed in terms of the LECs $c_{1}$, $f_{1}$ and $f_{2}$ [14:

$$
\delta_{\epsilon}=\frac{\mu_{c}}{8 \pi M_{\pi^{+}} F_{\pi}^{2} \mathcal{A}_{\text {str }}}\left[8 c_{1}\left(M_{\pi^{+}}^{2}-M_{\pi^{0}}^{2}\right)-e^{2}\left(4 f_{1}+f_{2}\right)\right]-2 \alpha \mu_{c}(\ln \alpha-1) \mathcal{A}_{s t r} .
$$

The quantity $c_{1}$ is the strong LEC from the ChPT Lagrangian 8, 10] and $F_{\pi}=92.4 \mathrm{MeV}$ is the physical value of the pion decay constant [14]. In Ref. [1] we obtained $c_{1}=-1.2 \pm 0.1$ $\mathrm{GeV}^{-1}$ using the PCQM approach. Our prediction for $c_{1}$ is close to the value $c_{1}=-0.9 m_{N}^{-1}$ deduced from the $\pi N$ partial wave analysis KA84 using baryon chiral perturbation theory [10]. Substituting the central values for our couplings $f_{1}=-19.5 \mathrm{MeV}, f_{2}=-8.7 \mathrm{MeV}$ and $c_{1}=-1.2 \mathrm{GeV}^{-1}$ into Eq. (68), we get $\delta_{\epsilon}=-2.8 \cdot 10^{-2}$. Our estimate is comparable to a prediction based on a potential model for the $\pi N$ interaction [21]: $\delta_{\epsilon}=-2.1 \cdot 10^{-2}$. 


\section{SUMMARY AND CONCLUSION}

Our results can be summarized as follows. Starting from the perturbative chiral quark model (PCQM), which, by construction, includes confinement and in general a nonlinear pion-quark interaction respecting global chiral symmetry, we made a perturbative expansion of the Lagrangian with respect to $1 / F$. In the second order $O\left(1 / F^{2}\right)$ one gets an interaction as well as a contact term quadratic in the pion field. We then show that the PCQM, both on the Lagrangian level and for observables, is fully equivalent to a Weinberg type model with an axial-vector pion-quark coupling and the explicit Weinberg-Tomozawa (WT) term. This connection is based either on an unitary transformation of the quark fields, which leaves the unperturbed part of the Lagrangian unaltered, or by the explicit evaluation of observables, such as the low-energy $\pi N S$-wave scattering amplitude.

As an intermediate result we note that:

i) the PCQM has two Lagrangian manifestations, where, as a matter of calculational convenience, either version can be used in the perturbative evaluation.

ii) the full equivalence between two model versions is only given, when the full quark propagator is introduced; hence a truncation of the intermediate-state sums will lead in general to different results.

iii) the connection between pseudoscalar and axial vector pion-quark coupling is made explicit; in the context of the PCQM this relation is established since the quarks remain on their energy-shell and quadratic terms in the pion field are kept consistently.

iv) the PCQM fulfils the low-energy constraints set by the WT result for $\pi N$ scattering lengths.

In a second step we extended the PCQM to include the electromagnetic field. Again, both Lagrangian versions are fully equivalent when introducing the photon field by minimal substitution. We apply the formalism to radiative corrections of low-energy nucleon observables. In the limit of a heavy nucleon mass we express the electromagnetic mass shift of nucleons and the $e^{2}$ corrections to the $\pi N$ amplitude in terms of the proton charge form factor only. Final expressions can be shown to be independent of the gauge choice. The result for the mass shift corrections is consistent with the model-independent one obtained in Ref. [18]. We can determine the complete set of the $O\left(p^{2}\right)$ electromagnetic low-energy couplings (LECs) $f_{1}, f_{2}$ and $f_{3}$ of the chiral perturbation theory (ChPT) effective Lagrangian. The magnitude of $f_{2}$ and its relation to $f_{1}$ and $f_{3}$ are obtained from an analysis of the nucleon electromagnetic mass shift and the leading radiative corrections to the $\pi N$ scattering amplitude at threshold. Using our values for $f_{1}$ and $f_{2}$ we also predict the isospin-breaking correction to the strong energy shift of the $\pi^{-} p$ atom in the $1 s$ state. This prediction is extremely important for the ongoing experiment "Pionic Hydrogen" at PSI, which plans to measure the ground-state shift and width of pionic hydrogen $\left(\pi^{-} p\right.$-atom) at the $1 \%$ level [22].

Acknowledgements. We thank A. Rusetsky for useful discussions. This work was supported by the DFG (grant FA67/25-1) and by the DAAD-PROCOPE project. 


\section{REFERENCES}

[1] V. E. Lyubovitskij, T. Gutsche, A. Faessler, and E.G. Drukarev, Phys. Rev. D 63, 54026 (2001); V. E. Lyubovitskij, T. Gutsche, and A. Faessler, hep-ph/0105043, Phys. Rev. C (in print).

[2] T. Gutsche and D. Robson, Phys. Lett. B 229, 333 (1989); T. Gutsche, Ph. D. Thesis, Florida State University, 1987 (unpublished).

[3] A. W. Thomas, Adv. Nucl. Phys. 13, 1 (1984); E. Oset, R. Tegen, and W. Weise; Nucl. Phys. A 426, 456 (1984); S. A. Chin, Nucl. Phys. A 382, 355 (1982).

[4] S. Weinberg, Phys. Rev. Lett. 17, 616 (1966).

[5] Y. Tomozawa, Nuovo Cim. A 46, 707 (1966).

[6] S. Weinberg, Phys. Rev. 166, 1568 (1968).

[7] J. Gasser, M. E. Sainio, and A. B. Švarc, Nucl. Phys. B 307, 779 (1988).

[8] N. Fettes, Ulf-G. Meissner, and S. Steininger, Nucl. Phys. A 640, 199 (1998).

[9] G. Müller and Ulf-G. Meissner, Nucl. Phys. B 556, 265 (1999);

N. Fettes, Ulf-G. Meissner, and S. Steininger, Phys. Lett. B 451, 233 (1999).

[10] T. Becher and H. Leutwyler, Eur. Phys. J. C 9, 643 (1999); JHEP 0106, 017 (2001).

[11] A. W. Thomas, J. Phys. G 7, L283 (1981); M. A. Morgan, G. A. Miller, and A. W. Thomas, Phys. Rev. D 33, 817 (1986).

[12] B. K. Jennings and O. V. Maxwell, Nucl. Phys. A 422, 589 (1984).

[13] M. Gell-Mann and M. Lévy, Nuovo Cim. 16, 1729 (1960).

[14] V. E. Lyubovitskij and A. Rusetsky, Phys. Lett. B 494, 9 (2000).

[15] N. N. Bogoliubov and D. V. Shirkov, An Introduction to Theory of Quantized Fields (Wiley-Interscience, New York, 1959).

[16] F. E. Close, An Introduction to Quarks and Partons (Academic Press, New York, 1979).

[17] M. Gell-Mann and F. Low, Phys. Rev. 84, 350 (1951).

[18] J. Gasser and H. Leutwyler, Phys. Rep. 87, 77 (1982).

[19] I. Duck, Phys. Lett. B 77, 223 (1978).

[20] S. Deser, M.L. Goldberger, K. Baumann, and W. Thirring, Phys. Rev. 96, 774 (1954).

[21] D. Sigg, A. Badertscher, P.F.A. Goudsmit, H.J. Leisi, and G.C. Oades, Nucl. Phys. A 609, 310 (1996).

[22] D. Gotta, $\pi N$ Newslett. 15, 276 (1999). 


\section{FIGURES}

FIG.1: Diagrams contributing to the $\pi N$ scattering amplitude: $s$-channel pole (1a), $u$ channel pole (1b) and seagull diagram (1c).

FIG.2: Diagrams contributing to the electromagnetic mass shift of the nucleon: one-body (2a) and two-body diagram (2b).

FIG.3: Diagrams contributing to the leading $e^{2} / F^{2}$ radiative corrections to the $\pi N$ amplitude at threshold. 


\section{FIGURES}

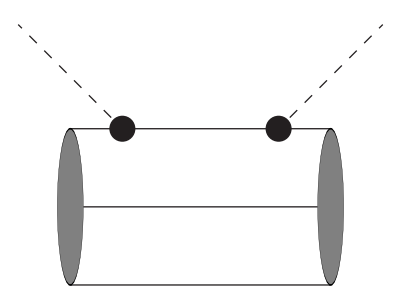

(a)

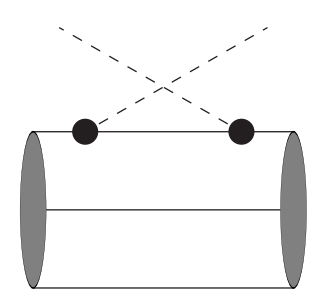

(b)

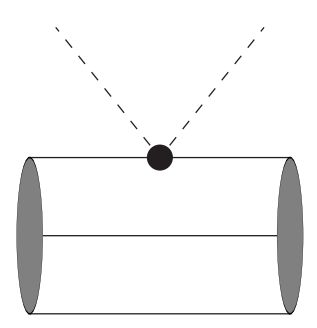

(c)

Fig.1

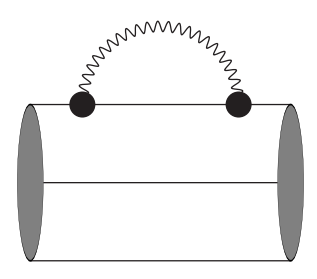

(a)

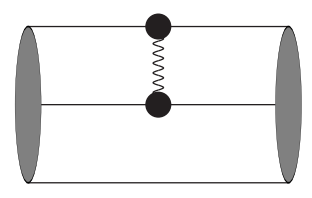

(b)

Fig.2 


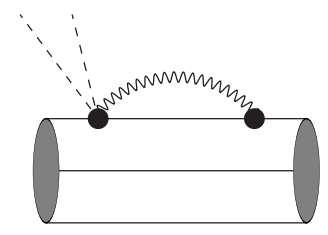

(a)

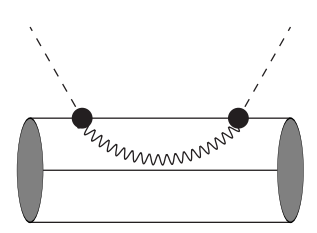

(d)

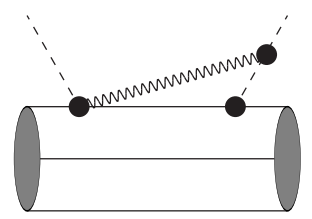

(g)

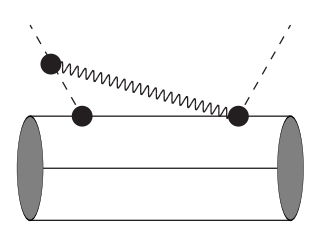

(j)

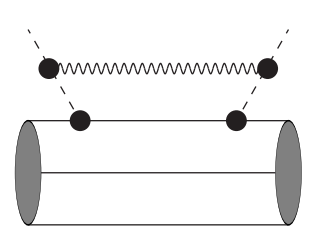

(m)

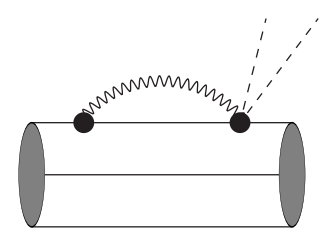

(b)

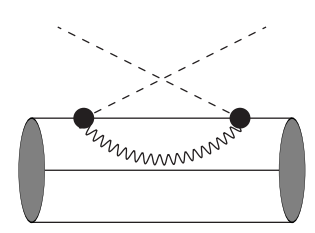

(e)

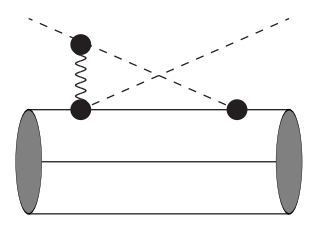

(h)

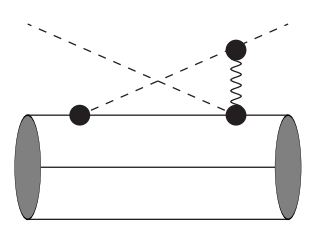

(k)

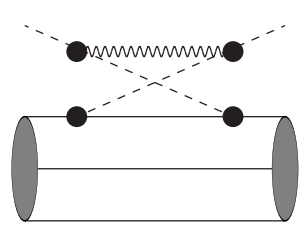

(n)

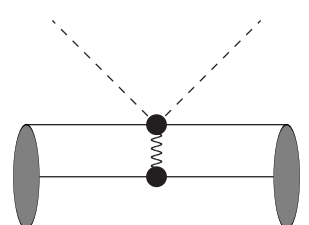

(c)

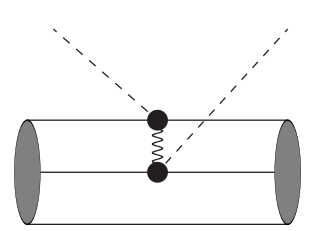

(f)

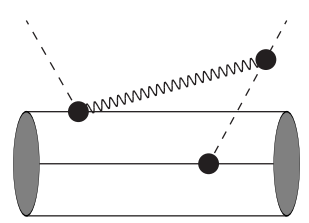

(i)

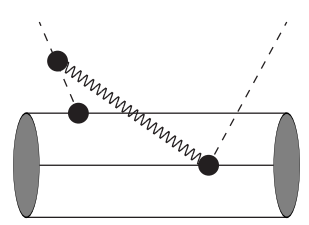

(1)

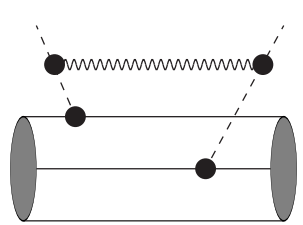

(o)

Fig.3 